\title{
Interval Estimation Methods for Discrete-time Linear Time-invariant Systems
}

\author{
Wentao Tang, Zhenhua Wang, Ye Wang, Tarek Raïssi, and Yi Shen Member, IEEE
}

\begin{abstract}
This paper investigates interval estimation methods for discrete-time linear time-invariant systems. We propose a novel interval estimation method by integrating robust observer design with reachability analysis. By introducing $H_{\infty}$ design into interval estimation, the proposed method provides a systematic and effective approach to improve the accuracy of interval estimation. Moreover, the relationships and comparisons between the existing results and the proposed method are discussed in detail. Finally, simulation results are presented to demonstrate the effectiveness of the proposed method.
\end{abstract}

Index Terms-Interval estimation, set-membership, interval observer, reachability analysis.

\section{INTRODUCTION}

State estimation has been extensively investigated in the control community and widely used in various applications, such as feedback controller design, fault diagnosis and so on. In practice, state estimation may not converge to the real state due to the existence of model uncertainties. In general, state estimation methods can be classified into two categories: the stochastic and the deterministic ones [1]. The state estimation method based on stochastic theory assumes that the uncertainties have known probability distribution, for instance the Kalman filtering [2]. Although this assumption is suitable to deal with measurement noise, it may be less representative when modeling large disturbances mostly related to not wellknown deterministic behaviors [3]. The deterministic method, also known as the set-membership estimation, is based on a more general assumption that model uncertainties are unknown but bounded. Instead of estimating the state, a set enclosing the admissible values of state can be obtained under this assumption. In the literature, there are mainly two kinds of set-membership estimation methods: the first is based on set predictions/intersections and the second (known as interval observer) is based on the monotone system theory. So far, the set-membership estimation has been applied in a variety of fields such as biotechnological systems [4], [5], reachability analysis [6], [7] and fault diagnosis [8], [9].

The set-membership estimation method based on set predictions/intersections uses geometrical compact sets (e.g. ellipsoids and zonotopes) to bound model uncertainties [10] and

This work was supported by National Natural Science Foundation of China (Grant No. 61403104, 61773145). Corresponding author: Zhenhua Wang.

W. Tang, Z. Wang and Y. Shen are with School of Astronautics, Harbin Institute of Technology, Harbin 150001, P. R. China(email:tangwentao2015@hit.edu.cn;zhenhua.wang@hit.edu.cn).

Y. Wang is with Institut de Robótica i Informática Industrial (IRI), CSICUPC, Universitat Politécnica de Catalunya, C/. Llorens i Artigas 4-6,08028 Barcelona,Spain(e-mail:ywang@iri.upc.edu).

T. Raïssi is with the Conservatoire National des Arts et Mtiers (CNAM), 75141 Paris (e-mail: tarek.raissi@cnam.fr). then constructs compact sets to enclose all the admissible state values that are consistent with the measurements and the bounded uncertainties [11]. In the pioneering work of [12], a recursive set-membership estimation method based on ellipsoids was proposed. Since then, the set-membership estimation methods using ellipsoids has been studied by many authors [13]-[16]. However, for the ellipsoid-based method, the constructed sets can hardly enclose the admissible sets of state tightly. The set-membership estimation method based on the minimum-volume paralleltope was proposed in [17] to obtain more accurate estimation than that based on ellipsoids. Polytopes were also used to increase estimation accuracy [18], [19]. However, the method based on general polytopes has high computational cost and becomes numerically unstable with dimensions of the constructed sets growing large [20]. Compared with other geometrical sets, zonotopes, a special class of polytopes, can be used to achieve a good tradeoff between estimation accuracy and computation complexity. Basic operations for zonotopes can reduce to simple matrix calculations [3]. Zonotopes are shown to be suitable compact sets for controlling the wrapping effect [21], which refers to the additional growth of the constructed sets resulting from the propagations of overestimations at each step. The setmembership estimation method based on zonotopes has been extensively studied [1], [3], [11], [22]-[24]. Some criteria such as $P$-radius [23] and $F$-radius [3] to measure the size of zonotopes have been used to improve estimation accuracy.

Different from the method based on set predictions/intersections, the interval observer based on the monotone system theory consists of two sub-observers that provide the upper and lower bounds of the real state. Interval observer was first proposed in [4] and has received considerable attention recently [25]-[30]. The basic idea of this method is to design two sub-observers such that their estimation error dynamics are both cooperative and stable. The advantage of interval observer lies in its high computational efficiency. However, it is not a trivial work to construct a cooperative and stable error system, and even impossible for some dynamical systems. To overcome this limitation, the methods based on coordinate transformation have been proposed to relax the design condition of interval observer. The transformation matrix can be alternatively chosen to be time-varying [31] or constant by solving a Sylvester equation [32]. Note that most of the existing results on interval observer design focus on continuous-time systems and only a few results are available in the discrete-time domain [33]-[36].

The zonotope-based method and interval observer are both effective in interval estimation. However, these two methods 
still have conservatisms to some extent. The implementation of a zonotope-based method relies on the use of the reduction operator that encloses the admissible values of state in a zonotope with a fixed dimension. The reduction operator may cause conservatism due to the wrapping effect. More accurate estimation can be obtained by using higher dimensional zonotopes, but it will increase the computational burden at the same time. For interval observer design, the cooperative constraint may limit the estimation accuracy. Although this constraint can be relaxed by coordinate transformations, it may cause additional conservatism.

The interval estimations obtained by the above two methods both can be converted into the sum of the conventional state estimation and the approximated bounds of the estimation error. Inspired by this, we propose a novel interval estimation method to overcome the aforementioned drawbacks of the zonotope-based method and interval observers. The main contributions of this paper lie in the following aspects:

1) We propose a novel interval estimation method by combining conventional observer design with reachability analysis. This method is intuitive and effective in controlling the wrapping effect.

2) We use the $H_{\infty}$ technique to attenuate the effects of disturbances and noises, which provides a systematic and effective way to improve the accuracy of interval estimation. The design condition can be converted into solving a linear matrix inequality (LMI).

3) The relationships among the zonotope-based method, interval observers and the proposed method are discussed explicitly in theory. Moreover, we show that the interval estimation obtained by the proposed method is more accurate than those by the zonotope-based method and interval observers under the same initial conditions.

Finally, two simulation results are presented to demonstrate the effectiveness of the proposed method and to verify the relationships of the three methods.

\section{PRELIMINARIES AND PROBLEM FORMULATION}

The following standard notations are used throughout this paper. $\mathbb{R}^{n}$ and $\mathbb{R}^{m \times n}$ denote the $n$ and $m \times n$ dimensional Euclidean space, respectively. $I_{n}$ denotes identity matrix with dimensions of $n \times n$. For simplicity, 0 represents zero number, vector or matrix of appropriate dimensions in this paper. The comparison operators $\geq,>, \leq,<$ and the absolute value operator $|\cdot|$ on vectors and matrices are understood elementwise. For a matrix $A \in \mathbb{R}^{m \times n}, A^{+}=\max \{0, A\}$ and $A^{-}=A^{+}-A$, where the operator, max, is also understood elementwise. $A^{T}$ denotes the transpose of $A . \mathbb{R}_{+}^{n}=\{x \in$ $\left.\mathbb{R}^{n}: x \geq 0\right\}$ and $\mathbb{R}_{+}^{m \times n}=\left\{x \in \mathbb{R}^{m \times n}: x \geq 0\right\}$. For a real symmetric matrix $P \in \mathbb{R}^{n \times n}, P \succ 0(P \prec 0)$ indicates that $P$ is positive (negative) definite. For a vector $x \in \mathbb{R}^{n}$, we use $\|x\|$ to denote its Euclidean norm, i.e. $\|x\|=\sqrt{x^{T} x}$. For a discrete transfer function $G(z),\|G(z)\|_{\infty}$ denotes its $H_{\infty}$ norm, i.e. the maximum singular value of $G(z)$. In a symmetric block matrix, we use $*$ to represent a term that can be induced by symmetry.
We use $\oplus$ to denote the Minkowski sum of two sets $\mathbf{X}$ and $\mathbf{Y}$, i.e.

$$
\mathbf{X} \oplus \mathbf{Y}=\{x+y: x \in \mathbf{X}, y \in \mathbf{Y}\}
$$

and use $\bigoplus$ to denote the Minkowski sum of a series of sets, i.e.

$$
\bigoplus_{i=1}^{m} \mathbf{S}_{i}=\mathbf{S}_{1} \oplus \cdots \oplus \mathbf{S}_{m}
$$

For a set $\mathbf{X} \subseteq \mathbb{R}^{n}$, its linear image associated with a matrix $L \in \mathbb{R}^{m \times n}$ is denoted as

$$
L \mathbf{X}=\{L x: x \in \mathbf{X}\} .
$$

The following definitions and properties are essential in this paper.

Definition 1. An interval vector $\mathbf{I} \subset \mathbb{R}^{n}$, which is also referred to as a box, is defined as

$$
\mathbf{I}=\left\{x: x \in \mathbb{R}^{n}, a_{i} \leq x_{i} \leq b_{i}, i=1, \ldots, n\right\} .
$$

For simplicity, we also denote $\mathbf{I}$ in (1) as

$$
\mathbf{I}=[a, b]
$$

where $a=\left[a_{1}, \ldots, a_{n}\right]^{T}$ and $b=\left[b_{1}, \ldots, b_{n}\right]^{T}$. And we denote the interval vector $\mathbf{I} \subset \mathbb{R}^{m}$ with all its components equal to $[-1,1]$ as $\mathbf{B}^{m}=[-1,1]^{m}$, which is also called a hypercube.

Property 1. For two interval vectors $[a, b] \subset \mathbb{R}^{n}$ and $[c, d] \subset$ $\mathbb{R}^{n}$, we have

$$
[a, b] \oplus[c, d]=[a+c, b+d] .
$$

Definition 2. For a set $\mathbf{S} \subset \mathbb{R}^{n}$, its interval hull is defined as the smallest interval vector containing it, which is denoted as

$$
\mathbf{S} \subseteq \operatorname{Box}(\mathbf{S})=[a, b]
$$

where $a=\left[a_{1}, \ldots, a_{n}\right]^{T}$ and $b=\left[b_{1}, \ldots, b_{n}\right]^{T}$. The smallest interval vector means that for any vector $s \in \mathbf{S}, a_{i} \leq s_{i} \leq$ $b_{i}, i=1, \ldots, n$ and $\left[a_{i}, b_{i}\right]$ is the smallest interval containing $s_{i}$.

Property 2. For a series of sets $\mathbf{S}_{i} \subset \mathbb{R}^{n}, i=1, \ldots, m$, the following equation holds

$$
\operatorname{Box}\left(\bigoplus_{i=1}^{m} \mathbf{S}_{i}\right)=\bigoplus_{i=1}^{m} \operatorname{Box}\left(\mathbf{S}_{i}\right) \text {. }
$$

Definition 3. An $m$-order zonotope $\mathcal{Z} \subset \mathbb{R}^{n}$ is an affine transformation of a hypercube $\mathbf{B}^{m}$, which is defined as

$$
\mathcal{Z}=\langle p, H\rangle=\left\{p+H z: z \in \mathbf{B}^{m}\right\},
$$

where $p \in \mathbb{R}^{n}$ is the center of $\mathcal{Z}$ and $H \in \mathbb{R}^{n \times m}$ is called the generator matrix of $\mathcal{Z}$, which determines the shape and volume of $\mathcal{Z}$.

Property 3. For zonotopes, the following equations hold:

$$
\begin{aligned}
\left\langle p_{1}, H_{1}\right\rangle \oplus\left\langle p_{2}, H_{2}\right\rangle & =\left\langle p_{1}+p_{2},\left[\begin{array}{ll}
H_{1} & H_{2}
\end{array}\right]\right\rangle, \\
L\langle p, H\rangle & =\langle L p, L H\rangle, \\
\langle p, H\rangle & \subseteq\langle p, \bar{H}\rangle
\end{aligned}
$$


where $p, p_{1}, p_{2} \in \mathbb{R}^{n}, H, H_{1}, H_{2} \in \mathbb{R}^{n \times m}$ and $L \in \mathbb{R}^{l \times n}$. $\bar{H}$ is a diagonal matrix with

$$
\bar{H}_{i, i}=\sum_{j=1}^{m}\left|H_{i, j}\right|, \quad i=1, \ldots, n .
$$

Property 4. For an $m$-order zonotope $\mathcal{Z}=\langle p, H\rangle \subset \mathbb{R}^{n}$, the components of $\operatorname{Box}(\mathcal{Z})=[a, b]$ can be obtained by

$$
\begin{cases}a_{i}=p_{i}-\sum_{j=0}^{m}\left|H_{i, j}\right|, & i=1, \ldots, n \\ b_{i}=p_{i}+\sum_{j=0}^{m}\left|H_{i, j}\right|, & i=1, \ldots, n\end{cases}
$$

The following lemma is also useful in the sequel development.

Lemma 1 ( [37]): Given a scalar $\gamma>0$, the discrete-time system described by

$$
\left\{\begin{array}{l}
x_{k+1}=\mathcal{A} x_{k}+\mathcal{B} u_{k} \\
y_{k}=\mathcal{C} x_{k}+\mathcal{D} u_{k}
\end{array}\right.
$$

is stable and its transfer function $G(z)=\mathcal{C}(z I-\mathcal{A})^{-1} \mathcal{B}+\mathcal{D}$ satisfies $\|G(z)\|_{\infty}<\gamma$, if and only if there exists a matrix $P \succ 0$ such that

$$
\left[\begin{array}{cc}
\mathcal{A}^{T} P \mathcal{A}+\mathcal{C}^{T} \mathcal{C}-P & * \\
\mathcal{B}^{T} P \mathcal{A}+\mathcal{D}^{T} \mathcal{C} & \mathcal{B}^{T} P \mathcal{B}+\mathcal{D}^{T} \mathcal{D}-\gamma^{2} I
\end{array}\right] \prec 0 .
$$

In this paper, we consider the following system:

$$
\left\{\begin{array}{l}
x_{k+1}=A x_{k}+B u_{k}+E w_{k} \\
y_{k}=C x_{k}+F v_{k}
\end{array}\right.
$$

where $x_{k} \in \mathbb{R}^{n_{x}}, u_{k} \in \mathbb{R}^{n_{u}}$ and $y_{k} \in \mathbb{R}^{n_{y}}$ denote the vectors of state, input and measurement output, respectively. $A \in \mathbb{R}^{n_{x} \times n_{x}}, B \in \mathbb{R}^{n_{x} \times n_{u}}, E \in \mathbb{R}^{n_{x} \times n_{w}}, C \in \mathbb{R}^{n_{y} \times n_{x}}$ and $F \in \mathbb{R}^{n_{y} \times n_{v}}$ are known matrices. $w_{k} \in \mathbb{R}^{n_{w}}$ and $v_{k} \in \mathbb{R}^{n_{v}}$ denote the vectors of unknown disturbance and measurement noise. Without loss of generality, $x_{0}, w_{k}$ and $v_{k}$ are assumed to be unknown but bounded as follows

$$
\left|x_{0}-p_{0}\right| \leq \bar{x}, \quad\left|w_{k}\right| \leq \bar{w}, \quad\left|v_{k}\right| \leq \bar{v}
$$

where $p_{0} \in \mathbb{R}^{n_{x}}, \bar{x} \in \mathbb{R}^{n_{x}}, \bar{w} \in \mathbb{R}^{n_{w}}$ and $\bar{v} \in \mathbb{R}^{n_{v}}$ are known vectors. According to Definition 3, (9) can be reformulated as

$$
x_{0} \in\left\langle p_{0}, H_{0}\right\rangle, \quad w_{k} \in\left\langle 0, D_{w}\right\rangle, \quad v_{k} \in\left\langle 0, D_{v}\right\rangle,
$$

where $H_{0}, D_{w}$ and $D_{v}$ are diagonal matrices with their diagonal elements equal to $\bar{x}, \bar{w}$ and $\bar{v}$, respectively. For simplicity, we denote $\mathbf{W}=\left\langle 0, D_{w}\right\rangle$ and $\mathbf{V}=\left\langle 0, D_{v}\right\rangle$. In the sequel, we will also use $\underline{w}=-\bar{w}$ and $\underline{v}=-\bar{v}$.

The objective of the interval estimation method is to obtain an interval vector $\left[\underline{x}_{k}, \bar{x}_{k}\right]$, which contains the real state $x_{k}$, i.e.

$$
\underline{x}_{k} \leq x_{k} \leq \bar{x}_{k}, \quad k \geq 0 .
$$

In this paper, we study three kinds of interval estimation methods for the system (8): (i) the method based on reachability analysis; (ii) the zonotope-based method; (iii) interval observers. Additionally, the comparisons and relationships among these three methods are discussed in detail.

\section{INTERVAL ESTIMATION BASED ON REACHABILITY ANALYSIS}

In this section, we propose a novel interval estimation method by integrating the robust observer design with the reachability analysis technique [6]. First, a Luenberger observer for system (8) is designed based on the $H_{\infty}$ technique such that its estimation error is robust against model uncertainties. Based on the designed observer, we obtain the interval estimation of state via reachability analysis.

Consider the following observer for the system (8):

$$
\hat{x}_{k+1}=A \hat{x}_{k}+B u_{k}+L\left(y_{k}-C \hat{x}_{k}\right),
$$

where $\hat{x}_{k}$ is the estimation of $x_{k}$ and $L \in \mathbb{R}^{n_{x} \times n_{y}}$ is the observer gain to be designed.

Define the estimation error as

$$
e_{k}=x_{k}-\hat{x}_{k},
$$

it follows that

$$
x_{k}=\hat{x}_{k}+e_{k} .
$$

If we can obtain an interval vector $\left[\underline{e}_{k}, \bar{e}_{k}\right]$ satisfying $\underline{e}_{k} \leq$ $e_{k} \leq \bar{e}_{k}$. Then, from (12), we have

$$
\hat{x}_{k}+\underline{e}_{k} \leq x_{k} \leq \hat{x}_{k}+\bar{e}_{k} .
$$

Therefore, the interval estimation of $x_{k}$ can be obtained as

$$
\left\{\begin{array}{l}
\bar{x}_{k}=\hat{x}_{k}+\bar{e}_{k} \\
\underline{x}_{k}=\hat{x}_{k}+\underline{e}_{k}
\end{array}\right.
$$

In order to obtain accurate estimation, $H_{\infty}$ technique is used to attenuate the effects of disturbance and noise on the estimation error.

To this end, we first analyze the error dynamics of the observer (10). By subtracting (10) from (8), the error dynamic system can be obtained as

$$
e_{k+1}=(A-L C) e_{k}+E w_{k}-L F v_{k} .
$$

It can be rewritten as

$$
e_{k+1}=A_{e} e_{k}+B_{e} d_{k}
$$

where $A_{e}=A-L C, B_{e}=\left[\begin{array}{ll}E & -L F\end{array}\right]$ and $d_{k}=$ $\left[\begin{array}{cc}w_{k}^{T} & v_{k}^{T}\end{array}\right]^{T}$.

For the error system (15), we proposed the following theorem to design $L$ such that the estimation error is robust against disturbance and noise.

Theorem 1: Given a scalar $\gamma>0$, if there exist a positive definite matrix $P \in \mathbb{R}^{n_{x} \times n_{x}}$ and a matrix $Y \in \mathbb{R}^{n_{x} \times n_{y}}$ such that

$$
\left[\begin{array}{cccc}
I_{n_{x}}-P & * & * & * \\
0 & -\gamma^{2} I_{n_{w}} & * & * \\
0 & 0 & -\gamma^{2} I_{n_{v}} & * \\
P A-Y C & P E & -Y F & -P
\end{array}\right] \prec 0
$$

and let $L=P^{-1} Y$, then system (15) is stable and the transfer function from $d_{k}$ to $e_{k}, G_{e d}(z)=\left(z I_{n_{x}}-A_{e}\right)^{-1} B_{e}$, satisfies $\left\|G_{e d}(z)\right\|_{\infty}<\gamma$.

Proof: Set $C_{e}=I_{n_{x}}$ and $D_{e}=0$, then according to Lemma 1, given a scalar $\gamma>0$, system (15) is stable 
and satisfies $\left\|G_{e d}(z)\right\|_{\infty}<\gamma$ if and only if there exists a symmetric matrix $P \succ 0$ such that

$$
\left[\begin{array}{cc}
A_{e}^{T} P A_{e}+I_{n_{x}}-P & * \\
B_{e}^{T} P A_{e} & B_{e}^{T} P B_{e}-\gamma^{2} I_{n_{w}+n_{v}}
\end{array}\right] \prec 0,
$$

which can be reformulated as

$$
\left[\begin{array}{cc}
I_{n_{x}}-P & * \\
0 & -\gamma^{2} I_{n_{w}+n_{v}}
\end{array}\right]+\left[\begin{array}{c}
A_{e}^{T} \\
B_{e}^{T}
\end{array}\right] P\left[\begin{array}{ll}
A_{e} & B_{e}
\end{array}\right] \prec 0 .
$$

By using the Schur complement lemma [38], (18) is equivalent to

$$
\left[\begin{array}{ccc}
I_{n_{x}}-P & * & * \\
0 & -\gamma^{2} I_{n_{w}+n_{v}} & * \\
P A_{e} & P B_{e} & -P
\end{array}\right] \prec 0 .
$$

Substituting $A_{e}$ and $B_{e}$ into (19) gets

$$
\left[\begin{array}{cccc}
I_{n_{x}}-P & * & * & * \\
0 & -\gamma^{2} I_{n_{w}} & * & * \\
0 & 0 & -\gamma^{2} I_{n_{v}} & * \\
P A-P L C & P E & -P L F & -P
\end{array}\right] \prec 0 .
$$

By setting $Y=P L$, we obtain (16).

The minimal $\gamma$ can be obtained by solving the following optimization problem:

$$
\begin{aligned}
& \min \gamma^{2}, \\
& \text { s.t. }
\end{aligned}
$$

and the feasible solution gives the observer gain by $L=$ $P^{-1} Y$.

After the observer gain $L$ being determined, the interval estimation of $x_{k}$ can be obtained using the following theorem, which is based on reachability analysis of estimation error.

Theorem 2: For the system (8) and the observer (10), given $\hat{x}_{0}=p_{0}$, then $x_{k}$ is bounded by the interval estimation, $\left[\underline{x}_{k}, \bar{x}_{k}\right]$ obtained from (13), where $\bar{e}_{k}$ and $\underline{e}_{k}$ are determined by

$$
\begin{aligned}
{\left[\underline{e}_{k}, \bar{e}_{k}\right]=} & \operatorname{Box}\left((A-L C)^{k}\left\langle 0, H_{0}\right\rangle\right) \\
& \oplus \bigoplus_{i=0}^{k-1} \operatorname{Box}\left((A-L C)^{i} E \mathbf{W}\right) \\
& \oplus \bigoplus_{i=0}^{k-1} \operatorname{Box}\left((A-L C)^{i}(-L F \mathbf{V})\right),
\end{aligned}
$$

with

$$
\left[\underline{e}_{0}, \bar{e}_{0}\right]=\operatorname{Box}\left(\left\langle 0, H_{0}\right\rangle\right)
$$

Proof: From the error system (14), $e_{k}$ can be obtained by

$$
\begin{aligned}
e_{k}= & (A-L C)^{k} e_{0}+\sum_{i=0}^{k-1}(A-L C)^{i} E w_{k-1-i} \\
& +\sum_{i=0}^{k-1}(A-L C)^{i}\left(-L F v_{k-1-i}\right),
\end{aligned}
$$

Define the reachable set of $e_{k}$ as $\boldsymbol{\Omega}_{k}$. From (23), we can obtain $\Omega_{k}$ as follows

$$
\begin{aligned}
\boldsymbol{\Omega}_{k}= & (A-L C)^{k} \boldsymbol{\Omega}_{0} \oplus \bigoplus_{i=0}^{k-1}(A-L C)^{i} E \mathbf{W} \\
& \oplus \bigoplus_{i=0}^{k-1}(A-L C)^{i}(-L F \mathbf{V})
\end{aligned}
$$

From (24) and (3), the interval hull of $\Omega_{k}$ can be obtained by

$$
\begin{aligned}
\operatorname{Box}\left(\boldsymbol{\Omega}_{k}\right)= & \operatorname{Box}\left((A-L C)^{k} \boldsymbol{\Omega}_{0}\right) \\
& \oplus \bigoplus_{i=0}^{k-1} \operatorname{Box}\left((A-L C)^{i} E \mathbf{W}\right) \\
& \oplus \bigoplus_{i=0}^{k-1} \operatorname{Box}\left((A-L C)^{i}(-L F \mathbf{V})\right)
\end{aligned}
$$

In addition, Since $x_{0} \in\left\langle 0, H_{0}\right\rangle$, we have $e_{0} \in\left\langle 0, H_{0}\right\rangle$ when $\hat{x}_{0}=p_{0}$, which implies

$$
\boldsymbol{\Omega}_{0}=\left\langle 0, H_{0}\right\rangle
$$

Then, from (21) and (22), we have

$$
\left[\underline{e}_{k}, \bar{e}_{k}\right]=\operatorname{Box}\left(\boldsymbol{\Omega}_{k}\right), \quad k \geq 0 .
$$

Since $e_{k} \in \boldsymbol{\Omega}_{k} \subseteq \operatorname{Box}\left(\boldsymbol{\Omega}_{k}\right)$, it follows $e_{k} \in\left[\underline{e}_{k}, \bar{e}_{k}\right]$, i.e. $\underline{e}_{k} \leq e_{k} \leq \bar{e}_{k}$. Finally, from (13), we have $\underline{x}_{k} \leq x_{k} \leq \bar{x}_{k}$.

By using the structure of (21), we can implement Theorem 2 via an iterative algorithm as follows.

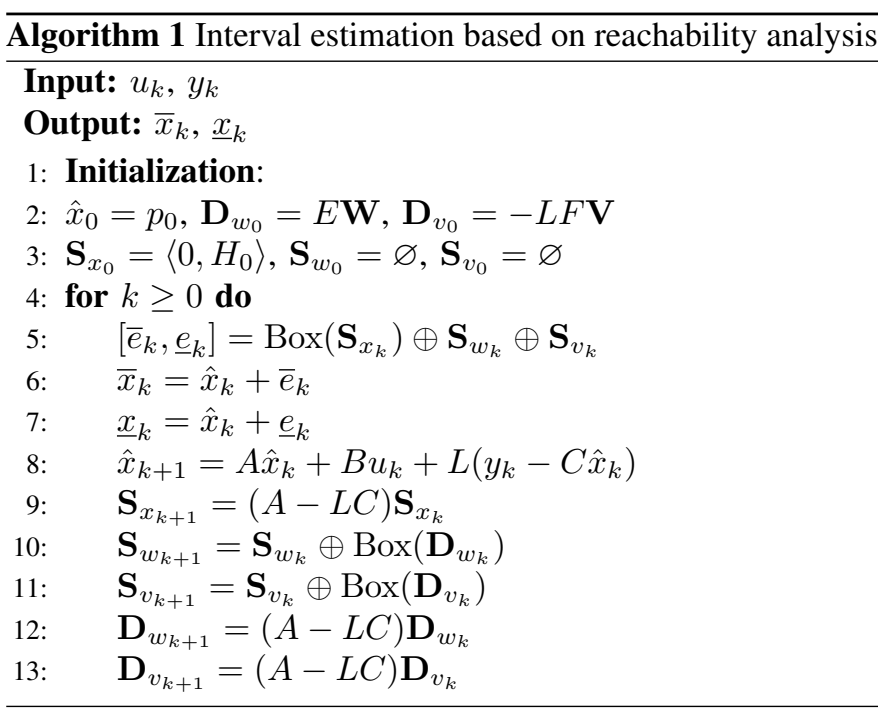

Remark 1. In Algorithm 1, over-approximations only exist in the $\operatorname{Box}(\cdot)$ operations. Note that the sets $\mathbf{S}_{x_{k}}, \mathbf{D}_{w_{k}}$ and $\mathbf{D}_{v_{k}}$ are both zonotopes and their interval hulls can be computed exactly by using Property 4. So Algorithm 1 is effective in controlling the wrapping effect. Besides, since the Minkowski sum of interval hulls can be converted into the sum of vectors according to (3), Algorithm 1 has high computational efficiency. 


\section{A. Boundedness of interval estimation}

When the real state $x_{k}$ is bounded, the boundedness of the interval estimation obtained from (13) is equivalent to the boundedness of the estimation error. Although Theorem 1 is proposed to attenuate the effects of disturbance and noise in the sense of $H_{\infty}$ norm, the observer gain obtained from Theorem 1 can guarantee the boundedness of the estimation error.

Theorem 3: Given a scalar $\gamma>0$, if there exist a positive definite matrix $P \in \mathbb{R}^{n_{x} \times n_{x}}$ and a matrix $Y \in \mathbb{R}^{n_{x} \times n_{y}}$ such that (16) holds and let $L=P^{-1} Y$, then there exist scalars $0<\alpha<1$ and $0<\beta<1$ such that

$$
\left\|e_{k}\right\|<\sqrt{\beta\left(\alpha^{k} V_{0}+\frac{1}{1-\alpha} \gamma^{2}\|d\|_{\infty}^{2}\right)}, \quad k \geq 1
$$

holds, where $V_{0}=e_{0}^{T} P e_{0}$ and $\|d\|_{\infty}^{2}=\sup _{k>0} d_{k}^{T} d_{k}$.

Proof: According to the proof of Theorem 1, if (16) holds and let $L=P^{-1} Y$, then (17) holds, which follows that

$$
\eta_{k}^{T}\left[\begin{array}{cc}
A_{e}^{T} P A_{e}+I_{n_{x}}-P & * \\
B_{e}^{T} P A_{e} & B_{e}^{T} P B_{e}-\gamma^{2} I_{n_{w}+n_{v}}
\end{array}\right] \eta_{k}<0,
$$

where $\eta_{k}=\left[\begin{array}{ll}e_{k}^{T} & d_{k}^{T}\end{array}\right]^{T}$. Then, we have

$$
\begin{aligned}
& e_{k}^{T} A_{e}^{T} P A_{e} e_{k}+e_{k}^{T} e_{k}-e_{k}^{T} P e_{k}+d_{k}^{T} B_{e}^{T} P A_{e} d_{k} \\
& \quad+e_{k}^{T} A_{e}^{T} P B_{e} d_{k}+d_{k}^{T} B_{e}^{T} P B_{e} d_{k}-\gamma^{2} d_{k}^{T} d_{k}<0
\end{aligned}
$$

For system (15), define the following Lyapunov function

$$
V_{k}=e_{k}^{T} P e_{k}, \quad k \geq 0,
$$

then (28) can be reformulated as

$$
V_{k+1}<V_{k}-e_{k}^{T} e_{k}+\gamma^{2} d_{k}^{T} d_{k}
$$

In addition, (16) implies $I_{n_{x}}-P \prec 0$, then there exist scalars $1<\alpha_{1}<\alpha_{2}$ such that

$$
\alpha_{1} I_{n_{x}} \prec P \prec \alpha_{2} I_{n_{x}} \text {. }
$$

It follows that $I_{n_{x}} \succ 1 / \alpha_{2} P$. Then from (29), we have

$$
\begin{aligned}
V_{k+1} & <V_{k}-e_{k}^{T} e_{k}+\gamma^{2}\|d\|_{\infty}^{2} \\
& <e_{k}^{T}\left(P-\frac{1}{\alpha_{2}} P\right) e_{k}+\gamma^{2}\|d\|_{\infty}^{2} \\
& <\left(1-\frac{1}{\alpha_{2}}\right) V_{k}+\gamma^{2}\|d\|_{\infty}^{2} .
\end{aligned}
$$

Define $\alpha=1-1 / \alpha_{2}$, it follows that $0<\alpha<1$. Then from (31), we have

$$
\begin{aligned}
V_{k} & <\alpha^{k} V_{0}+\sum_{i=0}^{k-1} \alpha^{i} \gamma^{2}\|d\|_{\infty}^{2} \\
& =\alpha^{k} V_{0}+\frac{1-\alpha^{k}}{1-\alpha} \gamma^{2}\|d\|_{\infty}^{2} \\
& <\alpha^{k} V_{0}+\frac{1}{1-\alpha} \gamma^{2}\|d\|_{\infty}^{2}
\end{aligned}
$$

In addition, (30) also implies that $\alpha_{1} e_{k}^{T} e_{k}<e_{k}^{T} P e_{k}=V_{k}$. Then from (32), we have

$$
\begin{aligned}
\left\|e_{k}\right\| & <\sqrt{\frac{1}{\alpha_{1}} V_{k}} \\
& <\sqrt{\frac{1}{\alpha_{1}}\left(\alpha^{k} V_{0}+\frac{1}{1-\alpha} \gamma^{2}\|d\|_{\infty}^{2}\right) .}
\end{aligned}
$$

Finally, by setting $\beta=1 / \alpha_{1}$, we gets (27).

\section{INTERVAL ESTIMATION BASED ON ZONOTOPES}

From (8), we have $y_{k}-C x_{k}-F v_{k}=0$. Then, the state vector $x_{k+1}$ can be obtained by

$$
x_{k+1}=A x_{k}+B u_{k}+E w_{k}+L\left(y_{k}-C x_{k}-F v_{k}\right),
$$

where $L \in \mathbb{R}^{n_{x} \times n_{y}}$. Based on (33), [3] gives a set-membership estimation method based on zonotopes, which can be described by the following proposition.

Proposition 1 ( [3]): For the system (8), if $x_{k} \in\left\langle p_{k}, H_{k}\right\rangle$, then the unknown state $x_{k+1}$ can be bounded in the zonotope $\left\langle p_{k+1}, H_{k+1}\right\rangle$ with

$$
\begin{aligned}
p_{k+1} & =(A-L C) p_{k}+B u_{k}+L y_{k}, \\
H_{k+1} & =\left[\begin{array}{lll}
(A-L C) \bar{H}_{k}, & E, & -L F
\end{array}\right],
\end{aligned}
$$

where $\bar{H}_{k}=\downarrow_{q, W} H_{k}$ is the reduction operation and $q \geq n_{x}$.

Denote $\operatorname{Box}\left(\left\langle p_{k}, H_{k}\right\rangle\right)=\left[\underline{x}_{k}^{z}, \bar{x}_{k}^{z}\right]$. From (5), it can be obtained by

$$
\begin{cases}\underline{x}_{k}^{z}(i)=p_{k}(i)-\sum_{i=0}^{s}\left|H_{k}(i, j)\right|, & i=1, \ldots, n_{x} \\ \bar{x}_{k}^{z}(i)=p_{k}(i)+\sum_{i=0}^{s}\left|H_{k}(i, j)\right|, & i=1, \ldots, n_{x}\end{cases}
$$

where $\underline{x}_{k}^{z}(i), \bar{x}_{k}^{z}(i)$ and $p_{k}(i)$ denote the i-th components of $\underline{x}_{k}^{z}, \bar{x}_{k}^{z}$ and $p_{k}$, respectively. $s$ is the column number of $H_{k}$. $H_{k}(i, j)$ denotes the element of $H_{k}$ in the i-th row and the $\mathrm{j}$-th column. The interval estimation based on zonotopes are obtained as $\bar{x}_{k}^{z}$ and $\underline{x}_{k}^{z}$, and we have $\underline{x}_{k}^{z} \leq x_{k} \leq \bar{x}_{k}^{z}$.

Remark 2. The column number of $H_{k}$ will increase linearly without the reduction operator. $\downarrow_{q, W} H_{k}$ obtains a new zonotope, $\left\langle 0, \bar{H}_{k}\right\rangle$ with fixed order $q$, such that $\left\langle 0, H_{k}\right\rangle \subseteq\left\langle 0, \bar{H}_{k}\right\rangle$. $W \in \mathbb{R}^{n_{x} \times n_{x}}$ is a positive definite matrix. $\downarrow_{q, W} H_{k}$ first reorders the columns of $H_{k}$ in decreasing weighted norm, $\left\|h_{i}\right\|_{W}=h_{i}^{T} W h_{i}, i=1, \ldots, s$, where $h_{i}$ is a column of $H_{k}$ and the obtained new matrix is denoted as $\tilde{H}$. Then, it replaces the last $s-q+n_{x}$ smallest columns of $\tilde{H}$ by a diagonal matrix $H^{-} \in \mathbb{R}^{n_{x} \times n_{x}}$ since the zonotope generated by these columns can be enclosed by a box. The whole process of $\downarrow_{q, W} H_{k}$ can be formulated as follows

$$
\begin{aligned}
& \tilde{H}=\left[h_{1}, \ldots, h_{s}\right], \quad\left\|h_{j}\right\|_{W} \geq\left\|h_{j+1}\right\|_{W} \\
& \text { if } s \leq q \\
& \quad \downarrow_{q, W} H_{k}=H_{k}, \\
& \text { else } \\
& \quad \downarrow_{q, W} H_{k}=\left[H^{+}, H^{-}\right], \\
& \text {where } H^{+}=\left[h_{1}, \ldots, h_{q-n_{x}}\right], \quad H_{i, i}^{-}=\sum_{j=q-n_{x}+1}^{s} \tilde{H}_{i, j} .
\end{aligned}
$$

\section{INTERVAL OBSERVER BASED ON MONOTONE SYSTEM} THEORY

\section{A. Interval observer without coordinate transformation}

The interval observer without coordinate transformation for the system (8) can be formulated as

$$
\left\{\begin{array}{l}
\bar{x}_{k+1}^{i}=A \bar{x}_{k}^{i}+B u_{k}+L\left(y_{k}-C \bar{x}_{k}^{i}\right)+\bar{d} \\
\underline{x}_{k+1}^{i}=A \underline{x}_{k}^{i}+B u_{k}+L\left(y_{k}-C \underline{x}_{k}^{i}\right)+\underline{d}
\end{array}\right.
$$


where $\bar{x}_{k}^{i}$ and $\underline{x}_{k}^{i}$ are the estimated upper and lower bounds of $x_{k}$, respectively. $\bar{d}$ and $\underline{d}$ are given as

$$
\left\{\begin{array}{l}
\bar{d}=E^{+} \bar{w}-E^{-} \underline{w}+(-L F)^{+} \bar{v}-(-L F)^{-} \underline{v} \\
\underline{d}=E^{+} \underline{w}-E^{-} \bar{w}+(-L F)^{+} \underline{v}-(-L F)^{-} \bar{v}
\end{array}\right.
$$

where $L \in \mathbb{R}^{n_{x} \times n_{y}}$ is the observer gain to be designed.

The interval observer (36) aims to estimate the upper and lower bounds of $x_{k}$, such that $\underline{x}_{k}^{i} \leq x_{k} \leq \bar{x}_{k}^{i}$. The following lemmas will be used in designing an interval observer based on the monotone system theory.

Lemma 2 ( [26]): For a vector $x \in \mathbb{R}^{n}$ and a matrix $\mathcal{A} \in$ $\mathbb{R}^{m \times n}$, if there exist $\underline{x}, \bar{x} \in \mathbb{R}^{n}$ such that $\underline{x} \leq x \leq \bar{x}$, then

$$
\mathcal{A}^{+} \underline{x}-\mathcal{A}^{-} \bar{x} \leq \mathcal{A} x \leq \mathcal{A}^{+} \bar{x}-\mathcal{A}^{-} \underline{x} .
$$

Lemma 3 ( [39]): Any solution of the system

$$
x_{k+1}=\mathcal{A} x_{k}+w_{k}
$$

with $w_{k} \in \mathbb{R}_{+}^{n}$ and $\mathcal{A} \in \mathbb{R}_{+}^{n \times n}$, is elementwise nonnegative for all $k \geq 0$ provided $x_{0} \geq 0$.

Based on Lemma 2 and Lemma 3, the conditions of designing the interval observer (36) can be described by the following theorem.

Theorem 4: For system (8), $\bar{x}_{k}^{i}$ and $\underline{x}_{k}^{i}$ in (36) satisfy $\underline{x}_{k}^{i} \leq$ $x_{k} \leq \bar{x}_{k}^{i}, \forall k \geq 0$, if $A-L C$ is nonnegative and $\underline{x}_{0}^{i} \leq x_{0} \leq \bar{x}_{0}^{i}$.

Proof: Define the interval estimation error as

$$
\left\{\begin{array}{l}
\bar{e}_{k}^{i}=\bar{x}_{k}^{i}-x_{k} \\
\underline{e}_{k}^{i}=\underline{x}_{k}^{i}-x_{k}
\end{array}\right.
$$

and it follows that

$$
\left\{\begin{array}{l}
\bar{x}_{k}^{i}=x_{k}+\bar{e}_{k}^{i} \\
\underline{x}_{k}^{i}=x_{k}+\underline{e}_{k}^{i}
\end{array}\right.
$$

From (38), $\underline{x}_{0}^{i} \leq x_{0} \leq \bar{x}_{0}^{i}$ implies $\bar{e}_{0}^{i} \geq 0$ and $\underline{e}_{0}^{i} \leq 0$. By subtracting (8) from (36), the interval estimation error dynamics can be formulated as

$$
\left\{\begin{array}{l}
\bar{e}_{k+1}^{i}=(A-L C) \bar{e}_{k}^{i}-E w_{k}+L F v_{k}+\bar{d} \\
\underline{e}_{k+1}^{i}=(A-L C) \underline{e}_{k}^{i}-E w_{k}+L F v_{k}+\underline{d}
\end{array}\right.
$$

In addition, by using Lemma 2, (37) implies

$$
\left\{\begin{array}{l}
-E w_{k}+L F v_{k}+\bar{d}=\bar{d}-\left(E w_{k}-L F v_{k}\right) \geq 0 \\
-E w_{k}+L F v_{k}+\underline{d}=\underline{d}-\left(E w_{k}-L F v_{k}\right) \leq 0
\end{array}\right.
$$

Then according to Lemma $3, \underline{e}_{k}^{i} \leq 0$ and $\bar{e}_{k}^{i} \geq 0$ hold for all $k \geq 0$ when $A-L C$ is nonnegative. Then from (39), we have $\underline{x}_{k}^{i} \leq x_{k} \leq \bar{x}_{k}^{i}$.

To guarantee the boundedness of $\bar{e}_{k}^{i}$ and $\underline{e}_{k}^{i}$, the observer gain $L$ should be designed such that $A-L C$ is both nonnegative and Schur.

In [39], the observer gain $L$ is obtained by solving the following LMIs:

$$
\begin{gathered}
{\left[\begin{array}{cc}
P & P A-Y C, \\
A^{T} P-C^{T} Y^{T} & P
\end{array}\right] \succ 0,} \\
P \succ 0 \\
P A-Y C \geq 0,
\end{gathered}
$$

where $P \in \mathbb{R}^{n_{x} \times n_{x}}$ is a diagonal matrix, $Y \in \mathbb{R}^{n_{x} \times n_{y}}$ and the feasible solution gives $L=P^{-1} Y$.

\section{B. Interval observer based on coordinate transformation}

It is not a trivial work to design an observer with cooperative and stable error system. To overcome this difficulty, the interval observer based on coordinate transformation has been proposed to obtain more relaxed design conditions, which has no requirement for the cooperativity of the error system.

An interval observer based on coordinate transformation can be described by the following theorem, which is modified from Theorem 2 in [33].

Theorem 5: If there exist a matrix $L \in \mathbb{R}^{n_{x} \times n_{y}}$ and a invertible matrix $S \in \mathbb{R}^{n_{x} \times n_{x}}$ such that $A-L C$ is Schur and $R=S(A-L C) S^{-1}$ is nonnegative, then

$$
\underline{x}_{k}^{t} \leq x_{k} \leq \bar{x}_{k}^{t}, \quad \forall k \geq 0
$$

provided $\underline{x}_{0}^{t} \leq x_{0} \leq \bar{x}_{0}^{t}$, where $\underline{x}_{k}^{t}$ and $\bar{x}_{k}^{t}, k \geq 1$, are obtained by the following process:

$$
\begin{aligned}
\bar{z}_{0} & =S^{+} \bar{x}_{0}^{t}-S^{-} \underline{x}_{0}^{t}, \\
\underline{z}_{0} & =S^{+} \underline{x}_{0}^{t}-S^{-} \bar{x}_{0}^{t}, \\
\bar{z}_{k+1} & =R \bar{z}_{k}+S B u_{k}+S L y_{k}+\bar{g}, \\
\underline{z}_{k+1} & =R \underline{z}_{k}+S B u_{k}+S L y_{k}+\underline{g}, \\
\bar{x}_{k+1}^{t} & =\left(S^{-1}\right)^{+} \bar{z}_{k+1}-\left(S^{-1}\right)^{-} \underline{z}_{k+1}, \\
\underline{x}_{k+1}^{t} & =\left(S^{-1}\right)^{+} \underline{z}_{k+1}-\left(S^{-1}\right)^{-} \bar{z}_{k+1},
\end{aligned}
$$

with

$$
\left\{\begin{array}{l}
\bar{g}=(S E)^{+} \bar{w}-(S E)^{-} \underline{w}+(-S L F)^{+} \bar{v}-(-S L F)^{-} \underline{v} \\
\underline{g}=(S E)^{+} \underline{w}-(S E)^{-} \bar{w}+(-S L F)^{+} \underline{v}-(-S L F)^{-} \bar{v}
\end{array}\right.
$$

Proof: For the system (8), consider the coordinate transformation $z_{k}=S x_{k}$, then a new system can be obtained as follows

$$
\left\{\begin{array}{l}
z_{k+1}=S A S^{-1} z_{k}+S B u_{k}+S E w_{k} \\
y_{k}=C S^{-1} z_{k}+F v_{k}
\end{array}\right.
$$

Define

$$
\left\{\begin{array}{l}
\bar{e}_{k}^{z}=\bar{z}_{k}-z_{k} \\
\underline{e}_{k}^{z}=\underline{z}_{k}-z_{k}
\end{array}\right.
$$

and it follows that

$$
\left\{\begin{array}{l}
\bar{z}_{k}=z_{k}+\bar{e}_{k}^{z} \\
\underline{z}_{k}=z_{k}+\underline{e}_{k}^{z}
\end{array}\right.
$$

By combining (42c), (42d) and (44), the error dynamics of $\bar{e}_{k}^{z}$ and $\underline{e}_{k}^{z}$ can be obtained as follows

$$
\left\{\begin{array}{l}
\bar{e}_{k+1}^{z}=R \bar{e}_{k}^{z}-S E w_{k}+S L F v_{k}+\bar{g} \\
\underline{e}_{k+1}^{z}=R \underline{e}_{k}^{z}-S E w_{k}+S L F v_{k}+\underline{g}
\end{array}\right.
$$

Since $z_{0}=S x_{0}$, then according to Lemma 2, (42a) and (42b) imply $\underline{z}_{0} \leq z_{0} \leq \bar{z}_{0}$. And it follows that $\bar{e}_{0}^{z}=\bar{z}_{0}-z_{0} \geq 0$ and $\underline{e}_{0}^{z}=\underline{z}_{0}-z_{0} \leq 0$. In addition, by using Lemma 2, (43) implies

$$
\left\{\begin{array}{l}
-S E w_{k}+S L F v_{k}+\bar{g}=\bar{g}-\left(S E w_{k}-S L F v_{k}\right) \geq 0 \\
-S E w_{k}+S L F v_{k}+\underline{g}=\underline{g}-\left(S E w_{k}-S L F v_{k}\right) \leq 0
\end{array}\right.
$$

Then, according to Lemma $3, \bar{e}_{k}^{z} \geq 0$ and $\underline{e}_{k}^{z} \leq 0$ when $R$ is nonnegative. Consequently, from (46), we have $\underline{z}_{k} \leq z_{k} \leq \bar{z}_{k}$.

Since $z_{k}=S x_{k}$, we have $x_{k}=S^{-1} z_{k}$. Then according to Lemma 2, (42e) and (42f) imply $\underline{x}_{k}^{t} \leq x_{k} \leq \bar{x}_{k}^{t}$. 
To find the coordinate transformation matrix $S$ such that $R=S(A-L C) S^{-1}$ is nonnegative, the following lemma is first introduced.

Lemma 4 ( [33]): Given matrices $A \in \mathbb{R}^{n \times n}, L \in \mathbb{R}^{n \times m}$, $C \in \mathbb{R}^{m \times n}$ and $R \in \mathbb{R}^{n \times n}$ that has the same eigenvalues with $A-L C$, if there exist two vectors $e_{1} \in \mathbb{R}^{n}$ and $e_{2} \in$ $\mathbb{R}^{n}$ such that the pairs $\left(A-L C, e_{1}\right)$ and $\left(R, e_{2}\right)$ are both observable, then there exists a matrix $S \in \mathbb{R}^{n \times n}$ such that $R=S(A-L C) S^{-1}$.

In [32], $R$ is chosen as a nonnegative matrix whose diagonal elements equal the eigenvalues of $A-L C$ and the coordinate transformation matrix is obtained by

$$
S=O_{R}^{-1} O_{A-L C},
$$

where $O_{R}$ and $O_{A-L C}$ are the observable matrices of the pairs $\left(R, e_{2}\right)$ and $\left(A-L C, e_{1}\right)$.

\section{COMPARISONS AND RELATIONSHIPS AMONG THREE INTERVAL ESTIMATION METHODS}

The interval estimations obtained by the zonotope-based method and interval observers both can be converted into the sum of the state estimation of a conventional observer and the approximated bounds of its estimation error. Since Algorithm 1 is effective in controlling the wrapping effect, the interval estimation obtained by Algorithm 1 can be more accurate than those obtained by the zonotope-based method and interval observers.

\section{A. Comparison between the proposed method and the zonotope-based method}

Theorem 6: For system (8), with the same observer gain $L$ and the initial conditions that $\hat{x}_{0}=p_{0}$ and $\boldsymbol{\Omega}_{0}=\left\langle 0, H_{0}\right\rangle$, the following inequalities

$$
\left\{\begin{array}{l}
\bar{x}_{k}^{z} \geq \bar{x}_{k} \\
\underline{x}_{k}^{z} \leq \underline{x}_{k}
\end{array}\right.
$$

hold for the interval estimation obtained by Algorithm 1 and that by (34).

Proof: From (10) and (34a), we have $\hat{x}_{k}=p_{k}$ provided $\hat{x}_{0}=p_{0}$. Define $\boldsymbol{\Omega}_{k}^{h}=\left\langle 0, H_{k}\right\rangle$ and $\overline{\boldsymbol{\Omega}}_{k}^{h}=\left\langle 0, \bar{H}_{k}\right\rangle$. From (34b) and the properties of zonotopes (4), we have

$$
\begin{aligned}
\boldsymbol{\Omega}_{k+1}^{h} & =\left\langle 0, H_{k+1}\right\rangle \\
& =\left\langle 0,(A-L C) \bar{H}_{k}\right\rangle \oplus\langle 0, E\rangle \oplus\langle 0,-L F\rangle \\
& =(A-L C)\left\langle 0, \bar{H}_{k}\right\rangle \oplus E\left\langle 0, I_{n_{w}}\right\rangle \oplus(-L F)\left\langle 0, I_{n_{v}}\right\rangle \\
& =(A-L C) \overline{\mathbf{\Omega}}_{k}^{h} \oplus E \mathbf{W} \oplus(-L F \mathbf{V}) .
\end{aligned}
$$

Since $\overline{\boldsymbol{\Omega}}_{k}^{h} \supseteq \boldsymbol{\Omega}_{k}^{h}$, then from (50), we have

$$
\boldsymbol{\Omega}_{k+1}^{h} \supseteq(A-L C) \boldsymbol{\Omega}_{k}^{h} \oplus E \mathbf{W} \oplus(-L F \mathbf{V})
$$

Based on $\boldsymbol{\Omega}_{0}^{h}=\boldsymbol{\Omega}_{0}$, (24) and (51) imply

$$
\boldsymbol{\Omega}_{k}^{h} \supseteq \boldsymbol{\Omega}_{k}
$$

Denote $\operatorname{Box}\left(\boldsymbol{\Omega}_{k}^{h}\right)=\left[\underline{\omega}_{k}^{h}, \bar{\omega}_{k}^{h}\right]$. According to (5), we have

$$
\left\{\begin{array}{l}
\bar{\omega}_{k}^{h}=\sum_{i=0}^{s}\left|H_{k}(i, j)\right|, \quad i=1, \ldots, n_{x} \\
\underline{\omega}_{k}^{h}=-\sum_{i=0}^{s}\left|H_{k}(i, j)\right|, \quad i=1, \ldots, n_{x}
\end{array}\right.
$$

and consider (35), it follows that

$$
\left\{\begin{array}{l}
\bar{x}_{k}^{z}=p_{k}+\bar{\omega}_{k}^{h} \\
\underline{x}_{k}^{z}=p_{k}+\underline{\omega}_{k}^{h}
\end{array}\right.
$$

In addition, (52) implies

$$
\left\{\begin{array}{l}
\bar{\omega}_{k}^{h} \geq \bar{e}_{k} \\
\underline{\omega}_{k}^{h} \leq \underline{e}_{k}
\end{array}\right.
$$

Then, from (13), (53) and (54), it follows that (49) holds.

\section{B. Comparison between the proposed method and the interval} observer without coordinate transformation

The following lemma will be used to analyze the relationship between the proposed method and the interval observer without coordinate transformation.

Lemma 5: For an interval vector $\mathbf{I}=[a, b] \subset \mathbb{R}^{n}$ and a nonnegative matrix $A \in \mathbb{R}^{m \times n}$, the following equation holds

$$
\operatorname{Box}(A \mathbf{I})=A \mathbf{I}=[A a, A b] .
$$

Proof: Lemma 5 is a straight corollary of Lemma 2 since $A^{+}=A$ and $A^{-}=0$ when $A$ is a nonnegative matrix.

Theorem 7: For system (8),with the same observer gain $L$ and the initial conditions that $\bar{x}_{0}^{i}=\bar{x}_{0}$ and $\underline{x}_{0}^{i}=\underline{x}_{0}$, the following inequalities

$$
\left\{\begin{array}{l}
\bar{x}_{k}^{i} \geq \bar{x}_{k} \\
\underline{x}_{k}^{i} \leq \underline{x}_{k}
\end{array}\right.
$$

hold for the interval estimation obtained by Algorithm 1 and that by (36).

Proof: Define

$$
\left\{\begin{array}{l}
\widetilde{e}_{k}=\bar{x}_{k}^{i}-\hat{x}_{k} \\
e_{k}=\underline{x}_{k}^{i}-\hat{x}_{k}
\end{array}\right.
$$

and it follows that

$$
\left\{\begin{array}{l}
\bar{x}_{k}^{i}=\hat{x}_{k}+\widetilde{e}_{k} \\
\underline{x}_{k}^{i}=\hat{x}_{k}+\underline{e}_{k}
\end{array}\right.
$$

Subtracting (10) from (36) gets

$$
\left\{\begin{array}{l}
\widetilde{e}_{k+1}=(A-L C) \widetilde{e}_{k}+\bar{d} \\
e_{k+1}=(A-L C) \underline{e}_{k}+\underline{d}
\end{array}\right.
$$

then

$$
\left\{\begin{array}{l}
\widetilde{e}_{k}=(A-L C)^{k} \widetilde{e}_{0}+\sum_{i=0}^{k-1}(A-L C)^{i} \bar{d} \\
\widetilde{e}_{k}=(A-L C)^{k} \underline{e}_{0}+\sum_{i=0}^{k-1}(A-L C)^{i} \underline{d}
\end{array}\right.
$$

Define $\widetilde{\boldsymbol{\Omega}}_{k}$ as

$$
\begin{aligned}
\widetilde{\boldsymbol{\Omega}}_{k}= & \operatorname{Box}\left((A-L C)^{k} \operatorname{Box}\left(\boldsymbol{\Omega}_{0}\right)\right) \\
& \oplus \bigoplus_{i=0}^{k-1} \operatorname{Box}\left((A-L C)^{i} \operatorname{Box}(E \mathbf{W})\right) \\
& \oplus \bigoplus_{i=0}^{k-1} \operatorname{Box}\left((A-L C)^{i} \operatorname{Box}(-L F \mathbf{V})\right) .
\end{aligned}
$$

Since

$$
\begin{aligned}
& \operatorname{Box}\left(\boldsymbol{\Omega}_{0}\right) \supseteq \boldsymbol{\Omega}_{0}, \\
& \operatorname{Box}(E \mathbf{W}) \supseteq E \mathbf{W}, \\
& \operatorname{Box}(-L F \mathbf{V}) \supseteq-L F \mathbf{V},
\end{aligned}
$$


it follows that

$$
\begin{aligned}
& \operatorname{Box}\left((A-L C)^{k} \operatorname{Box}\left(\boldsymbol{\Omega}_{0}\right)\right) \supseteq \operatorname{Box}\left((A-L C)^{k} \boldsymbol{\Omega}_{0}\right), \\
& \bigoplus_{i=0}^{k-1} \operatorname{Box}\left((A-L C)^{i} \operatorname{Box}(E \mathbf{W})\right) \\
& \supseteq \bigoplus_{i=0}^{k-1} \operatorname{Box}\left((A-L C)^{i} E \mathbf{W}\right), \\
& \bigoplus_{i=0}^{k-1} \operatorname{Box}\left((A-L C)^{i} \operatorname{Box}(-L F \mathbf{V})\right) \\
& \supseteq \bigoplus_{i=0}^{k-1} \operatorname{Box}\left((A-L C)^{i}(-L F \mathbf{V})\right),
\end{aligned}
$$

then by comparing (58) with (25), we have $\widetilde{\boldsymbol{\Omega}}_{k} \supseteq \operatorname{Box}\left(\boldsymbol{\Omega}_{k}\right)$.

For the interval observer (36), $A-L C$ is nonnegative. Then according to Lemma 5 , the following equations hold.

$$
\begin{aligned}
& \operatorname{Box}\left((A-L C)^{k} \operatorname{Box}\left(\boldsymbol{\Omega}_{0}\right)\right)=(A-L C)^{k} \operatorname{Box}\left(\boldsymbol{\Omega}_{0}\right), \\
& \bigoplus_{i=0}^{k-1} \operatorname{Box}\left((A-L C)^{i} \operatorname{Box}(-E \mathbf{W})\right) \\
& =\bigoplus_{i=0}^{k-1}(A-L C)^{i} \operatorname{Box}(E \mathbf{W}), \\
& \bigoplus_{i=0}^{k-1} \operatorname{Box}\left((A-L C)^{i} \operatorname{Box}(-L F \mathbf{V})\right) \\
& =\bigoplus_{i=0}^{k-1}(A-L C)^{i} \operatorname{Box}(-L F \mathbf{V}) .
\end{aligned}
$$

In addition, by using Lemma 2, (37) implies that

$$
\underline{d} \leq E w_{k}-L F v_{k} \leq \bar{d}
$$

then we have

$$
\operatorname{Box}(E \mathbf{W} \oplus(-L F \mathbf{V}))=[\underline{d}, \bar{d}] .
$$

Combining (58), (59) and (60), $\widetilde{\Omega}_{k}$ can be reformulated as

$$
\begin{aligned}
\widetilde{\boldsymbol{\Omega}}_{k}= & (A-L C)^{k} \operatorname{Box}\left(\boldsymbol{\Omega}_{0}\right) \oplus \bigoplus_{i=0}^{k-1}(A-L C)^{i} \operatorname{Box}(E \mathbf{W}) \\
& \oplus \bigoplus_{i=0}^{k-1}(A-L C)^{i} \operatorname{Box}(-L F \mathbf{V}) \\
= & (A-L C)^{k} \operatorname{Box}\left(\boldsymbol{\Omega}_{0}\right) \\
& \oplus \bigoplus_{i=0}^{k-1}(A-L C)^{i}(\operatorname{Box}(E \mathbf{W}) \oplus \operatorname{Box}(-L F \mathbf{V})) \\
= & (A-L C)^{k} \operatorname{Box}\left(\boldsymbol{\Omega}_{0}\right) \\
& \oplus \bigoplus_{i=0}^{k-1}(A-L C)^{i} \operatorname{Box}(E \mathbf{W} \oplus(-L F \mathbf{V})) \\
= & (A-L C)^{k} \operatorname{Box}\left(\boldsymbol{\Omega}_{0}\right) \oplus \bigoplus_{i=0}^{k-1}(A-L C)^{i}[\underline{d}, \bar{d}] .
\end{aligned}
$$

Since $\operatorname{Box}\left(\boldsymbol{\Omega}_{0}\right)=\left[\underline{e}_{0}, \bar{e}_{0}\right]$ and $[\underline{d}, \bar{d}]$ are both interval vectors, then according to Lemma 5 and (61), $\widetilde{\Omega}_{k}$ is also an interval vector. Denote $\widetilde{\Omega}_{k}=\left[\underline{\omega}_{k}, \bar{\omega}_{k}\right]$, then by using Lemma 5 , we can obtain from (61) that

$$
\left\{\begin{array}{l}
\bar{\omega}_{k}=(A-L C)^{k} \bar{e}_{0}+\sum_{i=0}^{k-1}(A-L C)^{i} \bar{d} \\
\underline{\omega}_{k}=(A-L C)^{k} \underline{e}_{0}+\sum_{i=0}^{k-1}(A-L C)^{i} \underline{d}
\end{array}\right.
$$

Since $\bar{x}_{0}^{i}=\bar{x}_{0}$ and $\underline{x}_{0}^{i}=\underline{x}_{0}$, from (13) and (55), we have $\widetilde{e}_{0}=\bar{e}_{0}$ and $\underline{e}_{0}=\underline{e}_{0}$. Then from (57) and (62), it follows that $\bar{\omega}_{k}=\widetilde{e}_{k}$ and $\underline{\omega}_{k}=\underline{e}_{k}$. Since $\widetilde{\boldsymbol{\Omega}}_{k} \supseteq \operatorname{Box}\left(\boldsymbol{\Omega}_{k}\right)$, we have

$$
\left\{\begin{array}{l}
\bar{e}_{k} \leq \bar{\omega}_{k}=\widetilde{e}_{k} \\
\underline{e}_{k} \geq \underline{\omega}_{k}=\underline{e}_{k}
\end{array}\right.
$$

then in addition with (13) and (56), we have

$$
\left\{\begin{array}{l}
\bar{x}_{k}^{i}=\hat{x}_{k}+\widetilde{e}_{k} \geq \hat{x}_{k}+\bar{e}_{k}=\bar{x}_{k} \\
\underline{x}_{k}^{i}=\hat{x}_{k}+\underline{e}_{k} \leq \hat{x}_{k}+\underline{e}_{k}=\underline{x}_{k}
\end{array}\right.
$$

Remark 3. From the proof of Theorem 7, it can be inferred that the interval observer (36) can be converted into calculating the sum of the state estimation of the Luenberger observer (10) and the approximated bounds of the estimation error. Note that the interval observer (36) uses $\operatorname{Box}(E \mathbf{W} \oplus(-L F \mathbf{V}))$, which is an outer-approximation of $E \mathbf{W} \oplus(-L F \mathbf{V})$. Due to the wrapping effect, the interval estimation obtained by the interval observer (36) is also an outer-approximation of that obtained by Algorithm 1.

\section{Comparison between the proposed method and the interval observer based on coordinate transformation}

Although a stable but not cooperative system can be converted into a cooperative system via a change of coordinate, the coordinate transformation may cause large additional conservatism.

Theorem 8: For the system (8), with the same observer gain $L$ and the initial conditions that $\bar{x}_{0}^{t}=\bar{x}_{0}$ and $\underline{x}_{0}^{t}=\underline{x}_{0}$, the following inequalities

$$
\left\{\begin{array}{l}
\bar{x}_{k}^{t} \geq \bar{x}_{k} \\
\underline{x}_{k}^{t} \leq \underline{x}_{k}
\end{array}\right.
$$

hold for the interval estimation obtained by Algorithm 1 and that by (42).

Proof: For the system (44), consider the following observer:

$$
\hat{z}_{k+1}=S A S^{-1} \hat{z}_{k}+S B u_{k}+S L\left(y_{k}-C S^{-1} \hat{z}_{k}\right) .
$$

Pre-multiplying both sides of (64) by $S^{-1}$, we get

$$
S^{-1} \hat{z}_{k+1}=A S^{-1} \hat{z}_{k}+B u_{k}+L\left(y_{k}-C S^{-1} \hat{z}_{k}\right) .
$$

Let $\hat{z}_{0}=S \hat{x}_{0}$, it follows that $\hat{x}_{0}=S^{-1} \hat{z}_{0}$. Then, by comparing (65) with (10), it can be concluded that

$$
\hat{x}_{k}=S^{-1} \hat{z}_{k}
$$

and it follows that

$$
\hat{z}_{k}=S \hat{x}_{k}
$$

Define $e_{k}^{z}=z_{k}-\hat{z}_{k}$. Since $z_{k}=S x_{k}$, then from (11) and (67), we have

$$
e_{k}^{z}=S e_{k}
$$

Denote the reachable set of $e_{k}^{z}$ as $\boldsymbol{\Omega}_{k}^{z}$, then $\boldsymbol{\Omega}_{k}^{z}=S \boldsymbol{\Omega}_{k}$ according to (68). Denote $\operatorname{Box}\left(\boldsymbol{\Omega}_{k}^{z}\right)=\left[\underline{\omega}_{k}^{z}, \bar{\omega}_{k}^{z}\right]$, then

$$
\underline{\omega}_{k}^{z} \leq e_{k}^{z} \leq \bar{\omega}_{k}^{z}
$$

Note that $\left[\bar{z}_{k}, \underline{z}_{k}\right]$ is just the interval estimation obtained by the interval observer (36) for system (44) with replacing $x_{k}$ by $z_{k}, A$ by $S A S^{-1}, B$ by $S B, E$ by $S E, C$ by $C S^{-1}$ and $L$ by $S L$. Define

$$
\left\{\begin{array}{l}
\widetilde{e}_{k}^{z}=\bar{z}_{k}-\hat{z}_{k} \\
\underline{e}_{k}^{z}=\underline{z}_{k}-\hat{z}_{k}
\end{array}\right.
$$


and correspond $\left[\underline{\omega}_{k}^{z}, \bar{\omega}_{k}^{z}\right]$ and $\left[e_{k}^{z}, \widetilde{e}_{k}^{z}\right]$ to $\left[\underline{e}_{k}, \bar{e}_{k}\right]$ and $\left[e_{k}, \widetilde{e}_{k}\right]$, respectively, then the following inequalities hold according to (63)

$$
\left\{\begin{array}{l}
\widetilde{e}_{k}^{z} \geq \bar{\omega}_{k}^{z} \\
e_{k}^{z} \leq \underline{\omega}_{k}^{z}
\end{array}\right.
$$

and from (68) and (69), it follows that

$$
e_{k}^{z} \leq S e_{k} \leq \widetilde{e}_{k}^{z}
$$

Note that the set of $e_{k}$ described by (72) is a parallelotope and we denote it as $\widetilde{\mathbf{P}}$.

Define a new parallelotope $\mathbf{P}$ described by

$$
\underline{\omega}_{k}^{z} \leq S e_{k} \leq \bar{\omega}_{k}^{z}
$$

then from (71), we have $\mathbf{P} \subseteq \widetilde{\mathbf{P}}$. Since $\boldsymbol{\Omega}_{k}$ is the reachable set of $e_{k}$ which only contains the admissible values of $e_{k}$, we have

$$
\boldsymbol{\Omega}_{k} \subseteq \mathbf{P} \subseteq \widetilde{\mathbf{P}} .
$$

Let us recall that for $k \geq 1$,

$$
\left\{\begin{array}{l}
\bar{x}_{k}^{t}=\left(S^{-1}\right)^{+} \bar{z}_{k}-\left(S^{-1}\right)^{-} \underline{z}_{k} \\
\underline{x}_{k}^{t}=\left(S^{-1}\right)^{+} \underline{z}_{k}-\left(S^{-1}\right)^{-} \bar{z}_{k}
\end{array}\right.
$$

Define $\bar{e}_{k}^{t}=\bar{x}_{k}^{t}-\hat{x}_{k}$ and $\underline{e}_{k}^{t}=\underline{x}_{k}^{t}-\hat{x}_{k}$, it follows that

$$
\left\{\begin{array}{l}
\bar{x}_{k}^{t}=\hat{x}_{k}+\bar{e}_{k}^{t} \\
\underline{x}_{k}^{t}=\hat{x}_{k}+\underline{e}_{k}^{t}
\end{array}\right.
$$

From (66) and $S^{-1}=\left(S^{-1}\right)^{+}-\left(S^{-1}\right)^{-}$, we obtain

$$
\hat{x}_{k}=S^{-1} \hat{z}_{k}=\left(S^{-1}\right)^{+} \hat{z}_{k}-\left(S^{-1}\right)^{-} \hat{z}_{k}
$$

Subtract (77) from (75) and consider (70), we have

$$
\left\{\begin{array}{l}
\bar{e}_{k}^{t}=\left(S^{-1}\right)^{+} \widetilde{e}_{k}^{z}-\left(S^{-1}\right)^{-} e_{k}^{z} \\
\underline{e}_{k}^{t}=\left(S^{-1}\right)^{+} \underline{e}_{k}^{z}-\left(S^{-1}\right)^{-} \widetilde{e}_{k}^{z}
\end{array}\right.
$$

Then, according to Lemma 2 and consider $e_{k}=S^{-1}\left(S e_{k}\right)$, when $e_{k} \in \widetilde{\mathbf{P}}$ (i.e. (72) holds), it comes

$$
\underline{e}_{k}^{t} \leq e_{k} \leq \bar{e}_{k}^{t} \text {. }
$$

then we have

$$
\left[\underline{e}_{k}^{t}, \bar{e}_{k}^{t}\right]=\operatorname{Box}(\widetilde{\mathbf{P}}),
$$

In addition, from (74), we have

$$
\operatorname{Box}\left(\boldsymbol{\Omega}_{k}\right) \subseteq \operatorname{Box}(\widetilde{\mathbf{P}}),
$$

then from (26), (79) and (80), it follows that

$$
\left[\underline{e}_{k}, \bar{e}_{k}\right] \subseteq\left[\underline{e}_{k}^{t}, \bar{e}_{k}^{t}\right] .
$$

It is equivalent to

$$
\left\{\begin{array}{l}
\bar{e}_{k}^{t} \geq \bar{e}_{k} \\
\underline{e}_{k}^{t} \leq \underline{e}_{k}
\end{array}\right.
$$

Finally, from (13), (76) and (81), we have

$$
\left\{\begin{array}{l}
\bar{x}_{k}^{t}=\hat{x}_{k}+\bar{e}_{k}^{t} \geq \hat{x}_{k}+\bar{e}_{k}=\bar{x}_{k} \\
\underline{x}_{k}^{t}=\hat{x}_{k}+\underline{e}_{k}^{t} \leq \hat{x}_{k}+\underline{e}_{k}=\underline{x}_{k}
\end{array}\right.
$$

Remark 4. From the proof of Theorem 8, we note that there are two places introducing conservatism. The first one refers to (74) since $\widetilde{\mathbf{P}}$ is bigger than $\mathbf{P}$. The second one lies in (80). And the conservatism caused by (80) may be very large since the shape of $\boldsymbol{\Omega}_{k}$ could be far more complex than that of $\widetilde{\mathbf{P}}$.

\section{Simulations}

In this section, two examples are presented to demonstrate the effectiveness of the proposed method and to verify the relationships among the three interval estimation methods.

Example 1. We consider a DC servo-motor with a tacho generator and an incremental encoder described in [40]. The dynamics of this plant can be described by:

$$
\begin{aligned}
J \frac{\mathrm{d}^{2} \theta}{\mathrm{d} t^{2}}+\mu \frac{\mathrm{d} \theta}{\mathrm{d} t} & =K_{t} i \\
L \frac{\mathrm{d} i}{\mathrm{~d} t}+R i & =u-K_{e} \frac{\mathrm{d} \theta}{\mathrm{d} t}
\end{aligned}
$$

where $\theta$ is the motor position, $i$ and $u$ are the armature current and voltage, respectively. The parameters $J, \mu, K_{t}, L, R$ and $K_{e}$ are the motor inertia moment, frictional constant, torque constant, inductance, resistance and back EMF constant, respectively. After parameter identification, these parameters are obtained as $J=0.0985 \mathrm{Nms}^{2}, \mu=0.1482 \mathrm{Nms}, K_{t}=K_{e}=$ $0.4901 \mathrm{Vs}, L=1.3726 \mathrm{H}$ and $R=0.0062 \Omega$.

By defining the state $x=[\theta, n, i]^{T}$, where $n$ is the motor speed, the dynamics of this plant can be described by the following state-space model

$$
\dot{x}=A_{c} x+B_{c} u,
$$

where

$$
A_{c}=\left[\begin{array}{ccc}
0 & 1 & 0 \\
0 & -\frac{\mu}{J} & \frac{K_{t}}{J} \\
0 & -\frac{K_{e}}{L} & -\frac{R}{L}
\end{array}\right], \quad B_{c}=\left[\begin{array}{c}
0 \\
0 \\
\frac{1}{L}
\end{array}\right]
$$

and the subscript $c$ stands for continuous-time.

We use the Euler method to discretize the above continuoustime model with the sampling time $T_{s}=0.1 \mathrm{~s}$ and consider the measurements of the tacho generator and the incremental encoder. As a result, we obtain a discrete-time model in the form of (8) with

$$
\begin{gathered}
A=\left[\begin{array}{ccc}
1 & 0.1 & 0 \\
0 & 0.8495 & 0.4977 \\
0 & -0.0357 & 0.9995
\end{array}\right], \quad B=\left[\begin{array}{c}
0 \\
0 \\
0.0729
\end{array}\right], \\
C=\left[\begin{array}{lll}
1 & 0 & 0 \\
0 & 1 & 0
\end{array}\right], \quad E=I_{3}, \quad F=I_{2},
\end{gathered}
$$

The process disturbance $w_{k}$ encloses the fluctuation of input voltage, the errors caused by the parameter identification and the discretization. The process disturbance and the measurement noise are bounded as follows

$$
\left|w_{k}\right| \leq\left[\begin{array}{c}
0.0225 \\
0.0225 \\
0.0404
\end{array}\right], \quad\left|v_{k}\right| \leq\left[\begin{array}{l}
0.0564 \\
0.0564
\end{array}\right]
$$

In the simulation study, the initial state $x_{0}=$ $[0.0015 \mathrm{rad}, 0.0148 \mathrm{rad} / \mathrm{s}, 0 \mathrm{~A}]^{T}$ and the input $u=2 \mathrm{~V}$. The initial reachable set of $x_{0}$ are set as $p_{0}=[0,0,0]^{T}$ and $H_{0}=0.2 I_{3}$. Denote $L_{1}$ and $L_{2}$ as the observer gains obtained by solving the optimization problem (20) and the LMIs (41), respectively. They are as follows

$$
L_{1}=\left[\begin{array}{ll}
0.9998 & 0.1000 \\
0.0157 & 1.5371 \\
0.0315 & 1.3453
\end{array}\right], \quad L_{2}=\left[\begin{array}{cc}
0.7582 & 0.0784 \\
-2.5012 & 0.6764 \\
-0.0040 & -0.0360
\end{array}\right]
$$


The simulation results are shown in Fig.1-3. The asterisks are the values of $\theta, n$ and $i$. The solid lines represent the interval estimation obtained by Algorithm 1 and the dashdotted ones represent that by the zonotope-based method (34) with their observer gains both equal to $L_{1}$. The dashed lines and the dotted ones represent the interval estimations obtained by Algorithm 1 and the interval observer (36) with $L_{2}$. Fig.1-3 all illustrate that the interval estimation obtained by Algorithm 1 is more accurate that those obtained by the zonotope-based method (34) and the interval observer (36) under the same conditions. Moreover, we note that the interval estimations of $n$ and $i$ obtained with $L_{1}$ are much more accurate than those with $L_{2}$, which well demonstrates the effectiveness of the proposed method.

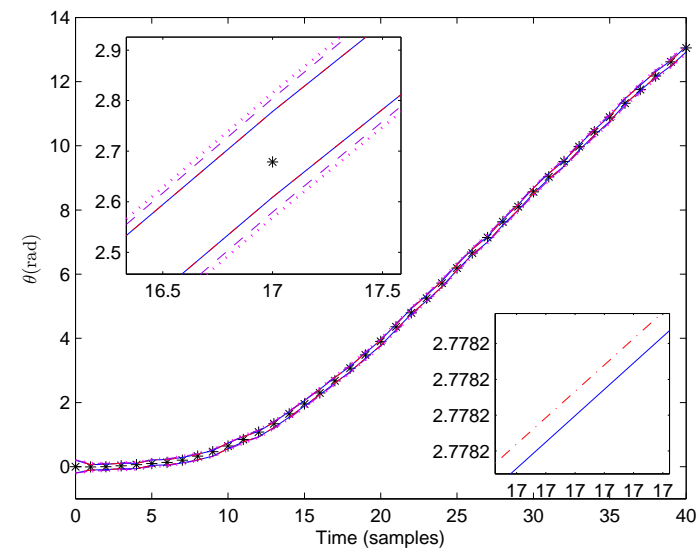

Fig. 1. Motor position $\theta$ and its interval estimations.

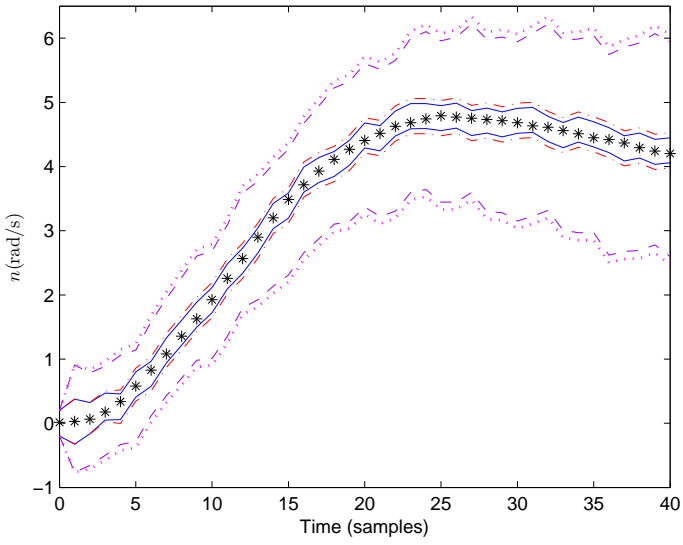

Fig. 2. Motor speed $n$ and its interval estimations.

Example 2. In order to illustrate the effect of coordinate transformation, consider the following numerical example in the form of (8) with

$$
\begin{aligned}
& A=\left[\begin{array}{cc}
1 & 0.5 \\
-0.5 & 1
\end{array}\right], \quad B=\left[\begin{array}{l}
0 \\
1
\end{array}\right], \quad C=\left[\begin{array}{ll}
0 & 1
\end{array}\right], \\
& E=I_{2}, \quad F=I_{2} .
\end{aligned}
$$

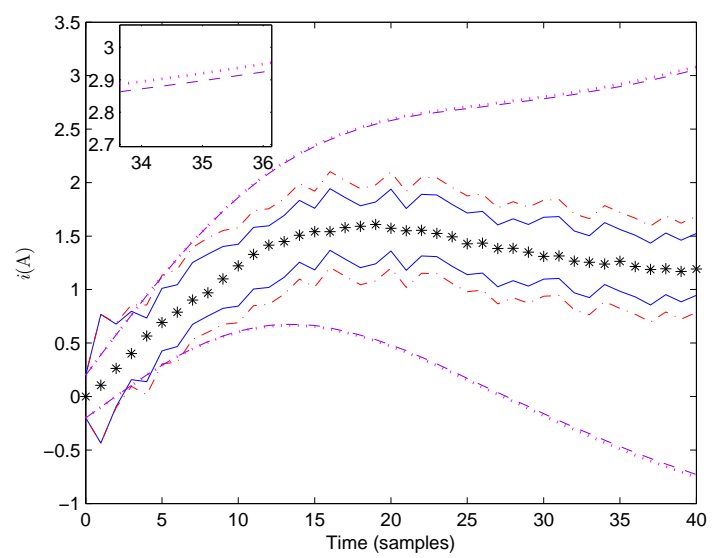

Fig. 3. Armature current $i$ and its interval estimations.

The disturbance and noise are bounded as follows

$$
\left|w_{k}\right| \leq\left[\begin{array}{l}
0.1 \\
0.1
\end{array}\right], \quad\left|v_{k}\right| \leq\left[\begin{array}{l}
0.1 \\
0.1
\end{array}\right] .
$$

There is no matrix $L \in \mathbb{R}^{2 \times 1}$ such that $A-L C$ is nonnegative. So the interval observer (36) does not exist. Solving the optimization problem in (20) yields

$$
L=\left[\begin{array}{c}
-0.8479 \\
1.6739
\end{array}\right]
$$

The eigenvalues of $A-L C$ is 0.3258 and 0.0003 . To design the interval observer based on coordinate transformation, $R$ is set as

$$
R=\left[\begin{array}{cc}
0.3258 & 0.2000 \\
0 & 0.0003
\end{array}\right]
$$

Using (48) with $e_{1}=\left[\begin{array}{ll}1 & 0\end{array}\right]^{T}$ and $e_{2}=\left[\begin{array}{ll}0.5 & -1\end{array}\right]^{T}$ gets

$$
S=\left[\begin{array}{ll}
5.1686 & 6.3352 \\
1.5843 & 3.1676
\end{array}\right]
$$

In the simulation study, $x_{0}=[1.2,0.9]^{T}$. The initial parameters of Algorithm 1 are set as $p_{0}=[1,1]^{T}$ and $H_{0}=0.5 I_{2}$. For the interval observer based on coordinate transformation, the initial interval estimations are set as $\bar{x}_{0}^{t}=\bar{x}_{0}=[1.5,1.5]^{T}$ and $\underline{x}^{t}=\underline{x}_{0}=[0.5,0.5]^{T}$.

The simulation results are depicted in Fig.4. Therein, the solid lines are the components of $x_{k}$, the dashed and dashdotted ones represent the interval estimations obtained by Algorithm 1 and those by (42), respectively. Fig. 4 shows that the interval estimations obtained by Algorithm 1 are much more accurate than those by the interval observer based on coordinate transformation.

To better verify Theorem 8 , Figure 5 is plotted with $k=40$, where the polytope with solid lines is a zonotope that represents $\boldsymbol{\Omega}_{k}$, the dash-dotted parallelogram is $\widetilde{\mathbf{P}}$ described by (72), the dashed rectangular is the interval vector $\left[\underline{e}_{k}, \bar{e}_{k}\right]$, the dotted rectangular is $\left[e_{k}, \widetilde{e}_{k}\right]$ and the dots are $e_{k}$ of 1000 independent experiments. It shows that all the dots of $e_{k}$ are enclosed in $\boldsymbol{\Omega}_{k}$ and $\left[\underline{e}_{k}, \bar{e}_{k}\right]$ is just $\operatorname{Box}\left(\boldsymbol{\Omega}_{k}\right)$. The shape of $\widetilde{\mathbf{P}}$ differs greatly from that of $\boldsymbol{\Omega}_{k}$, which causes large conservatism in the interval estimation obtained by (42). 

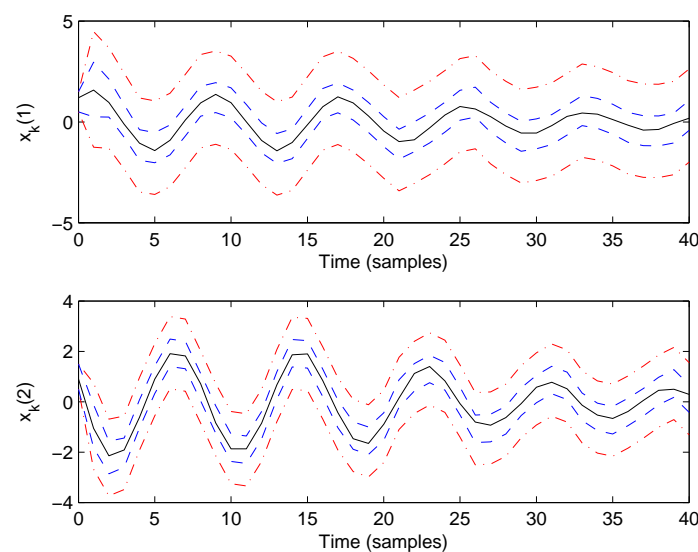

Fig. 4. $x_{k}$ and its interval estimations by Algorithm 1 and (42)

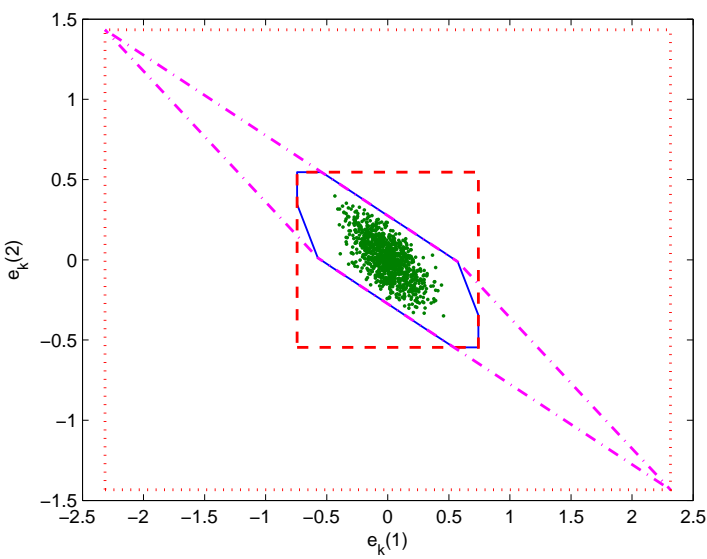

Fig. 5. Reachabililty analysis of the estimation error $e_{k}$.

\section{CONCLUSION}

This paper reviews several interval estimation methods for discrete-time linear time-invariant systems mainly including the zonotope-based method and interval observers. In view of that the interval estimation obtained by the two kinds of methods both can be converted into the sum of the state estimation and the approximated error bounds of a conventional observer, we propose a novel interval estimation method that combines the $H_{\infty}$ robust observer design with reachability analysis. The proposed method has high computational efficiency and can obtain more accurate interval estimation than the zonotopebased method and interval observers under the same conditions. Compared with interval observers, the proposed method overcomes the cooperative constraint and avoids the additional conservatism caused by coordinate transformation. The simulation results have demonstrated effectiveness of the proposed method and verified the relationships among the zonotopebased method, interval observers and the proposed method.

\section{REFERENCES}

[1] C. Combastel, "An extended zonotopic and Gaussian Kalman filter (EZGKF) merging set-membership and stochastic paradigms: toward non-linear filtering and fault detection," Annual Reviews in Control, vol. 42, pp. 232-243, 2016.
[2] R. E. Kalman, "A new approach to linear filtering and prediction problems," Journal of Basic Engineering, vol. 82, no. 1, pp. 35-45, 1960.

[3] C. Combastel, "Zonotopes and Kalman observers: gain optimality under distinct uncertainty paradigms and robust convergence," Automatica, vol. 55, pp. 265-273, 2015.

[4] J.-L. Gouzé, A. Rapaport, and M. Z. Hadj-Sadok, "Interval observers for uncertain biological systems," Ecological Modelling, vol. 133, no. 1, pp. 45-56, 2000.

[5] M. Moisan, O. Bernard, and J.-L. Gouzé, "Near optimal interval observers bundle for uncertain bioreactors," Automatica, vol. 45, no. 1 , pp. 291-295, 2009.

[6] A. Girard, C. Le Guernic, and O. Maler, "Efficient computation of reachable sets of linear time-invariant systems with inputs," in International Workshop on Hybrid Systems: Computation and Control. Springer, 2006, pp. 257-271.

[7] C. Le Guernic and A. Girard, "Reachability analysis of linear systems using support functions," Nonlinear Analysis: Hybrid Systems, vol. 4, no. 2, pp. 250-262, 2010.

[8] J. K. Scott, R. Findeisen, R. D. Braatz, and D. M. Raimondo, "Input design for guaranteed fault diagnosis using zonotopes," Automatica, vol. 50, no. 6, pp. 1580-1589, 2014.

[9] F. Xu, V. Puig, C. Ocampo-Martinez, S. Olaru, and F. Stoican, "Settheoretic methods in robust detection and isolation of sensor faults," International Journal of Systems Science, vol. 46, no. 13, pp. 23172334, 2015.

[10] C. Combastel, "A state bounding observer based on zonotopes," in European Control Conference. IEEE, 2003, pp. 2589-2594.

[11] T. Alamo, J. Bravo, and E. Camacho, "Guaranteed state estimation by zonotopes," Automatica, vol. 41, no. 6, pp. 1035-1043, 2005.

[12] F. Schweppe, "Recursive state estimation: unknown but bounded errors and system inputs," IEEE Transactions on Automatic Control, vol. 13, no. 1, pp. 22-28, 1968.

[13] E. Fogel and Y.-F. Huang, "On the value of information in system identification-bounded noise case," Automatica, vol. 18, no. 2, pp. 229 238, 1982.

[14] A. V. Savkin and I. R. Petersen, "Robust state estimation and model validation for discrete-time uncertain systems with a deterministic description of noise and uncertainty," Automatica, vol. 34, no. 2, pp 271-274, 1998.

[15] A. B. Kurzhanski and P. Varaiya, "Ellipsoidal techniques for reachability analysis: internal approximation," Systems \& Control letters, vol. 41, no. 3, pp. 201-211, 2000.

[16] F. Chernousko, "Ellipsoidal state estimation for dynamical systems," Nonlinear Analysis: Theory, Methods \& Applications, vol. 63, no. 5, pp. 872-879, 2005.

[17] L. Chisci, A. Garulli, and G. Zappa, "Recursive state bounding by parallelotopes," Automatica, vol. 32, no. 7, pp. 1049-1055, 1996.

[18] J. S. Shamma and K.-Y. Tu, "Set-valued observers and optimal disturbance rejection," IEEE Transactions on Automatic Control, vol. 44 no. 2, pp. 253-264, 1999.

[19] F. Blanchini and S. Miani, Set-theoretic methods in control. Springer, 2008.

[20] J. K. Scott, D. M. Raimondo, G. R. Marseglia, and R. D. Braatz, "Constrained zonotopes: a new tool for set-based estimation and fault detection," Automatica, vol. 69, pp. 126-136, 2016.

[21] W. Kühn, "Rigorously computed orbits of dynamical systems without the wrapping effect," Computing, vol. 61, no. 1, pp. 47-67, 1998.

[22] C. Combastel, "A state bounding observer for uncertain non-linear continuous-time systems based on zonotopes," in 44th IEEE Conference on Decision and Control and European Control Conference. IEEE, 2005, pp. 7228-7234.

[23] V. T. H. Le, C. Stoica, T. Alamo, E. F. Camacho, and D. Dumur, "Zonotopic guaranteed state estimation for uncertain systems," Automatica, vol. 49, no. 11, pp. 3418-3424, 2013.

[24] C. Combastel, "Merging Kalman filtering and zonotopic state bounding for robust fault detection under noisy environment," IFACPapersOnLine, vol. 48, no. 21, pp. 289-295, 2015.

[25] F. Mazenc and O. Bernard, "Asymptotically stable interval observers for planar systems with complex poles," IEEE Transactions on Automatic Control, vol. 55, no. 2, pp. 523-527, 2010.

[26] D. Efimov, T. Raïssi, S. Chebotarev, and A. Zolghadri, "Interval state observer for nonlinear time varying systems," Automatica, vol. 49, no. 1 , pp. 200-205, 2013.

[27] R. E. H. Thabet, T. Raïssi, C. Combastel, D. Efimov, and A. Zolghadri, "An effective method to interval observer design for time-varying systems," Automatica, vol. 50, no. 10, pp. 2677-2684, 2014. 
[28] Y. Wang, D. M. Bevly, and R. Rajamani, "Interval observer design for LPV systems with parametric uncertainty," Automatica, vol. 60, pp. 7985, 2015.

[29] F. Cacace, A. Germani, and C. Manes, "A new approach to design interval observers for linear systems," IEEE Transactions on Automatic Control, vol. 60, no. 6, pp. 1665-1670, 2015.

[30] G. Zheng, D. Efimov, F. J. Bejarano, W. Perruquetti, and H. Wang, "Interval observer for a class of uncertain nonlinear singular systems," Automatica, vol. 71, pp. 159-168, 2016.

[31] F. Mazenc and O. Bernard, "Interval observers for linear time-invariant systems with disturbances," Automatica, vol. 47, no. 1, pp. 140-147, 2011.

[32] T. Raïssi, D. Efimov, and A. Zolghadri, "Interval state estimation for a class of nonlinear systems," IEEE Transactions on Automatic Control, vol. 57, no. 1, pp. 260-265, 2012.

[33] D. Efimov, W. Perruquetti, T. Raïssi, and A. Zolghadri, "On interval observer design for time-invariant discrete-time systems," in $2013 \mathrm{Eu}$ ropean Control Conference. IEEE, 2013, pp. 2651-2656.

[34] D. Efimov, W. Perruquetti, T. Raïssi, and A. Zolghadri, "Interval observers for time-varying discrete-time systems," IEEE Transactions on Automatic Control, vol. 58, no. 12, pp. 3218-3224, 2013.

[35] F. Mazenc, T. N. Dinh, and S. I. Niculescu, "Interval observers for discrete-time systems," International Journal of Robust and Nonlinear Control, vol. 24, no. 17, pp. 2867-2890, 2014.

[36] E. Chambon, L. Burlion, and P. Apkarian, "Overview of LTI interval observer design: towards a non-smooth optimisation-based approach," IET Control Theory and Applications, vol. 10, pp. 1258-1268, 2016.

[37] S. Xu and J. Lam, Robust control and filtering of singular systems. Verlag, Berlin, Germany: Springer, 2006.

[38] S. Boyd, L. El Ghaoui, E. Feron, and V. Balakrishnan, Linear matrix inequalities in system and control theory. SIAM, 1994.

[39] D. Efimov and T. Raïssi, "Design of interval observers for uncertain dynamical systems," Automation and Remote Control, vol. 77, no. 2, pp. 191-225, 2016.

[40] M. Buciakowski, M. Witczak, M. Mrugalski, and D. Theilliol, "A quadratic boundedness approach to robust dc motor fault estimation," Control Engineering Practice, vol. 66, pp. 181-194, 2017. 